\title{
Helmholtz bright and boundary solitons
}

\author{
J M Christian ${ }^{1,3}$, G S McDonald ${ }^{1}$ and P Chamorro-Posada ${ }^{2}$ \\ 1 Joule Physics Laboratory, School of Computing, Science and Engineering, \\ Institute for Materials Research, University of Salford, Salford M5 4WT, UK \\ 2 Departmento de Teoría de la Señal y Comunicaciones e Ingeniería Telemática, \\ Universidad de Valladolid, ETSI Telecomunicación, Campus Miguel Delibes s/n, \\ 47011 Valladolid, Spain
}

\begin{abstract}
We report, for the first time, exact analytical boundary solitons of a generalized cubic-quintic Non-Linear Helmholtz (NLH) equation. These solutions have a linked-plateau topology that is distinct from conventional dark soliton solutions; their amplitude and intensity distributions are spatially delocalized and connect regions of finite and zero wave-field disturbances (suggesting also the classification as "edge solitons"). Extensive numerical simulations compare the stability properties of recentlyreported Helmholtz bright solitons, for this type of polynomial non-linearity, to those of the new boundary solitons. The latter are found to possess a remarkable stability characteristic, exhibiting robustness against perturbations that would otherwise lead to the destabilizing of their bright-soliton counterparts.
\end{abstract}

PACS number(s): $\quad$ 42.65.- $\mathrm{k}$ (nonlinear optics), 42.65.Tg (optical solitons), 05.45.Yv (solitons, nonlinear dynamics of)

Submitted to Journal of Physics A: Mathematical and Theoretical as a Paper.

Resubmitted on $20^{\text {th }}$ December 2006

\footnotetext{
${ }^{3}$ Corresponding author.
} 


\section{Introduction}

Solitons are ubiquitous entities in nature. Whenever linear effects (such as dispersion, diffraction or diffusion) are balanced exactly by non-linearity (self-phase modulation, self-focusing or reactionkinetic properties, respectively), robust self-trapped structures - solitons - can emerge as dominant modes of the system dynamics. These localized self-stabilizing non-linear waves arise widely in nature since quite different physical systems are governed by a relatively small set of universal equations, at least to first approximation. Solitons are often sech ("bell")- or tanh ("S")-shaped structures. The latter class are sometimes referred to as kink solitons, and they generally possess topologically non-trivial phase distributions.

Phase-topological kink solitons appear in a range of physically diverse systems, and play the role of "fronts" and "domain walls". In classical mechanics, for example, they describe collective long-wave excitations on a line of weakly-coupled pendula. In condensed matter, kink solitons arise in simple models of one-dimensional lattice-dynamics when studying the motion of dislocations and domain walls in ferromagnetic crystals, and they also play a key role in the phenomenological understanding of phase transitions. In chemical kinetics, kink solitons appear as solutions to reaction-diffusion equations. They also occur in hydrodynamics, plasma physics, quantum field theory and cosmology. Comprehensive reviews of these systems can be found in Refs. [1-4].

Our principle concern in this paper is with spatial soliton beams found in non-linear optics $[5,6]$. These types of soliton can arise when the tendency of a collimated light beam to diffract is opposed by the non-linear properties of the optical medium. When these two effects (diffractive broadening, and narrowing due to self-focusing) become comparable, then a stationary beam can exist whose transverse intensity distribution is invariant along the propagation direction. Spatial solitons are of theoretical interest as particular solutions to generic non-linear evolution equations, but they are also the subject of considerable experimental investigation. The robustness of these spatial beams against perturbations suggests their use as elementary units in future photonic systems, where they could play a central role in applications such as all-optical switching, beam steering, optical interconnects and image/information processing.

\section{Paraxial versus non-paraxial solitons}

In non-linear optics, one is often interested in planar waveguide geometry, where there is a reference longitudinal $(z)$ and a single effective transverse $(x)$ dimension. A simple model of beam propagation is provided by the universal Non-Linear Schrödinger (NLS) equation, which allows for the paraxial evolution of one-dimensional (transverse) diffraction and a Kerr non-linearity (where the refractive index varies linearly with the local beam intensity). The NLS equation is exactly integrable and its bright [7] and dark [8] soliton solutions are well known. The latter are tanh-type kink structures that possess nontrivial phase topology. Other, more general, NLS-type models have also been studied. These tend to account for more involved material properties, and allow for broader classes of refractive index dependencies while retaining the possibility of exact analytical soliton solutions. The most familiar generalizations are classic cubic-quintic [9-13] and the power-law [14,15] models. More complicated refractive-index distributions involve polynomial- [16-18] and saturable-type [19,20] non-linearities.

NLS-type equations provide an adequate description if the optical beams are: (i) much broader than their carrier wavelength, (ii) of sufficiently low intensity, and (iii) propagating along (or at nearnegligible angles with respect to) the reference axis. These criteria define the paraxial approximation. If all three conditions are not satisfied simultaneously, the beam is referred to as "non-paraxial". Nonparaxial beams have received much attention in the literature over the last three decades. Since the seminal work of Lax et al [21], a large body of research has considered contexts involving ultra-narrow beams, where condition (i) no longer holds [22-26]. As a consequence, the terms 'narrow beam' and 'non-paraxial beam' have, to a large extent, become interchangeable. However, this oversimplified interpretation omits the possibility of other distinct physical regimes of non-paraxiality, such as the propagation and multiplexing of broad beams at arbitrary angles with respect to the reference direction. This angular context, in which only condition (iii) is relaxed, defines Helmholtz non-paraxiality [27].

In other works, we demonstrated that oblique (off-axis) soliton evolution $[27,28]$ and soliton-soliton interactions [29] can be described by Non-Linear Helmholtz (NLH) equations. Since beams are always assumed to be broad, narrow-beam corrections [21-26] to the governing equation are not necessary. In NLH descriptions, the electric field may be regarded as effectively scalar (with a single transverse com- 
ponent orientated in the plane of the waveguide), and the refractive-index distributions may also be treated within the scalar approximation [23]. Models based upon the NLH formalism omit the slowlyvarying envelope (SVEA) approximation, and thus respect the rotational symmetry inherent to uniform media ( $x$ and $z$ appear symmetrically in the governing equation). Diffraction is therefore allowed in both spatial dimensions. $x-z$ equivalence also permits travelling- and standing-wave solutions, and allows description of beams that propagate and interact at any angle relative to the reference direction.

Exact analytical Helmholtz soliton solutions are known for focusing [27] and defocusing [30] Kerr media, and also for power-law [31] media. Vector generalizations of the Kerr solitons have also recently been derived [32]. A wide variety of exact analytical Helmholtz soliton solutions are now known for a wide variety of polynomial-type and saturable non-linearities [33]. These new nonparaxial families include hyperbolic (exponential) and algebraic solutions. Rigorous numerical simulations have verified that Helmholtz solitons are generally stable robust attractors [29-35].

In this paper, we derive a new family of spatially-extended solitons for a polynomial-type NLH equation. These new "boundary solitons" (or "edge solitons") possess a double-plateau structure in their amplitude, as opposed to their phase. This gives them a rare characteristic amongst non-linear optical waves since their intensity distributions share this delocalized feature. Linear stability analysis is considered for non-linear plane wave,s while full numerical simulations reveals remarkable stability characteristics for boundary solitons that could not have been predicted a priori. This enhanced stability may allow such non-linear waves to be exploited in future optical device applications.

\section{Helmholtz bright and boundary solitons}

\subsection{Model equation}

We consider a continuous-wave transverse-electric (TE) scalar field $\tilde{E}(x, z, t)$ with angular frequency $\omega$,

$$
\tilde{E}(x, z, t)=E(x, z) \exp (-i \omega t)+E^{*}(x, z) \exp (+i \omega t),
$$

that is assumed to be polarized in the $(x, z)$ plane of the waveguide. Since the complex spatial envelopes are assumed to vary on a scale much larger than the optical wavelength (as they must be for the scalar approximation to hold), the envelope $E(x, z)$ is governed by an NLH equation,

$$
\left(\frac{\partial^{2}}{\partial z^{2}}+\frac{\partial^{2}}{\partial x^{2}}\right) E(x, z)+\frac{\omega^{2}}{c^{2}} n^{2}\left(|E|^{2}\right) E(x, z)=0 .
$$

We consider a generalized polynomial-type scalar refractive-index distribution $n=n_{0}+n_{N L}$, where $n_{0}$ is the linear index at frequency $\omega, n_{N L}=n_{\sigma}|E|^{\sigma}-n_{2 \sigma}|E|^{2 \sigma}, n_{\sigma}$ and $n_{2 \sigma}$ are (positive) non-linear coefficients, and the exponent $\sigma>0$. This model can describe weak saturation effects in some planar waveguides [16,17]. For $n_{\sigma}|E|^{\sigma} \ll n_{0}$ and $n_{2 \sigma}|E|^{2 \sigma} \ll n_{0}$ one finds that, to a good approximation, $n^{2} \simeq n_{0}^{2}+2 n_{0} n_{\sigma}|E|^{\sigma}-2 n_{0} n_{2 \sigma}|E|^{2 \sigma}$ and thus equation (2) becomes

$$
\left(\frac{\partial^{2}}{\partial z^{2}}+\frac{\partial^{2}}{\partial x^{2}}\right) E(x, z)+\frac{\omega^{2}}{c^{2}}\left(n_{0}^{2}+2 n_{0} n_{\sigma}|E|^{\sigma}-2 n_{0} n_{2 \sigma}|E|^{2 \sigma}\right) E(x, z)=0 .
$$

With an appropriate rescaling, equation (3) may be expressed in the normalized form

$$
\kappa \frac{\partial^{2} u}{\partial \zeta^{2}}+\mathrm{i} \frac{\partial u}{\partial \zeta}+\frac{1}{2} \frac{\partial^{2} u}{\partial \xi^{2}}+\alpha|u|^{\sigma} u-\gamma|u|^{2 \sigma} u=0
$$


Here, $\zeta=z / L_{D}, \xi=\sqrt{2} x / w_{0}$ and $L_{D}=k w_{0}^{2} / 2$ is the diffraction length of a reference (paraxial) Gaussian beam of waist $w_{0}$. The (inverse) beam-width parameter is $\kappa=1 /\left(k w_{0}\right)^{2}=\left(\lambda / w_{0}\right)^{2} / 4 \pi^{2} n_{0}^{2}$. The wave-field $u$ is the normalized envelope of the complex electric field, $E(x, z)=E_{0} u(x, z) \exp (i k z)$, where $k=n_{0} k_{0}, k_{0}=2 \pi / \lambda$ and $\lambda$ is the optical wavelength. By choosing a particular scaling such as $E_{0}=\left(n_{0} / n_{\sigma} L_{D} k\right)^{1 / \sigma}$, one may set $\alpha=1$ and then $\gamma=E_{0}^{\sigma}\left(n_{2 \sigma} / n_{\sigma}\right)$. However, we retain maximum flexibility in our model by leaving the formal scaling unspecified and thereby derive exact analytical solutions to the most general model (4). The paraxial model corresponding to equation (4) can be recovered if and only if $\kappa \partial_{\zeta \zeta} u$ is negligible with respect to other terms [16,17]. The single smallparameter limit $\kappa \rightarrow 0$ is not, by itself, a sufficient condition for this recovery.

\subsection{Conservation laws}

Integrable models tend to possess a discrete infinity of conserved quantities $[7,8]$. In contrast, equation (4) is non-integrable so, at most, only a few integrals-of-motion can be defined [33]. Three such invariants correspond to the energy-flow $W$, the momentum $M$ and the Hamiltonian $H$ :

$$
\begin{aligned}
& W=\int_{-\infty}^{+\infty} d \xi\left[|u|^{2}-i \kappa\left(u^{*} \frac{\partial u}{\partial \zeta}-u \frac{\partial u^{*}}{\partial \zeta}\right)\right], \\
& M=\int_{-\infty}^{+\infty} d \xi\left[\frac{i}{2}\left(u^{*} \frac{\partial u}{\partial \xi}-u \frac{\partial u^{*}}{\partial \xi}\right)-\kappa\left(\frac{\partial u^{*}}{\partial \zeta} \frac{\partial u}{\partial \xi}+\frac{\partial u^{*}}{\partial \xi} \frac{\partial u}{\partial \zeta}\right)\right], \\
& H=\int_{-\infty}^{+\infty} d \xi\left[\frac{1}{2} \frac{\partial u^{*}}{\partial \xi} \frac{\partial u}{\partial \xi}-\kappa \frac{\partial u^{*}}{\partial \zeta} \frac{\partial u}{\partial \zeta}-\alpha \frac{|u|^{2+\sigma}}{1+\frac{1}{2} \sigma}+\gamma \frac{|u|^{2(1+\sigma)}}{1+\sigma}\right] .
\end{aligned}
$$

If an initial condition for equation (4) does not correspond to an exact solution, beam evolution typically involves the shedding of a small amount of radiation. Such radiation modes can be regarded as a dissipation mechanism that permits a perturbed solitary excitation to lose energy locally while total energy is conserved globally. That is, integrals (5) for the entire non-linear solution (which captures both solitary waves and radiative components) are still preserved. A dissipative interpretation of the localized system behaviour is also instructive because it allows the stability properties of various soliton families to be classified according to their phase-space portraits.

\subsection{Soliton solutions}

Since equation (4) retains the full spatially-symmetric (i.e. ' $2^{\text {nd }}$-order-in- $z$ ') character, it supports both forward- and backward-propagating solutions $[31,34,35]$. By convention, we consider here only the forward solutions and note that the corresponding paraxial model [16] has no counterpart to the backward solutions. An exact analytical bright soliton for equation (4) has recently been derived [33],

$$
u(\xi, \zeta)=\left[\frac{\eta}{\cosh [\Theta(\xi, \zeta)]+\Gamma}\right]^{\frac{1}{\sigma}} \exp \left[\mathrm{i} \sqrt{\frac{1+4 \kappa\left(\mu^{2} / 2 \sigma^{2}\right)}{1+2 \kappa V^{2}}}\left(-V \xi+\frac{\zeta}{2 \kappa}\right)\right] \exp \left(-\mathrm{i} \frac{\zeta}{2 \kappa}\right)
$$

where

$$
\Theta(\xi, \zeta)=\mu \frac{\xi+V \zeta}{\sqrt{1+2 \kappa V^{2}}}, \quad \eta \equiv\left(\frac{\mu^{2}}{2 \sigma^{2}}\right) \frac{2+\sigma}{\alpha} \Gamma, \quad \Gamma=\left[1-\left(\frac{\mu^{2}}{2 \sigma^{2}}\right) \frac{(2+\sigma)^{2}}{1+\sigma} \frac{\gamma}{\alpha^{2}}\right]^{-\frac{1}{2}} .
$$


$\eta$ and $V$ are amplitude and transverse velocity parameters, respectively. $\mu$ is a free parameter determining the soliton width, and the existence of the solution is guaranteed provided

$$
\mu<\mu_{C R}=\frac{\alpha \sigma}{2+\sigma} \sqrt{\frac{2(1+\sigma)}{\gamma}} .
$$

Here we report, for the first time, a boundary soliton solution of equation (4). An ansatz approach was used to seek the on-axis solution. Then, by exploiting the invariance relations of. (4) [34], an orthogonal transformation was deployed to derive the (more general) off-axis solution,

$$
\begin{aligned}
& u_{ \pm}(\xi, \zeta)=\left[\frac{\eta}{\exp [ \pm \Theta(\xi, \zeta)]+\Gamma}\right]^{\frac{1}{\sigma}} \exp \left[\mathrm{i} \sqrt{\frac{1+4 \kappa\left(\mu^{2} / 2 \sigma^{2}\right)}{1+2 \kappa V^{2}}}\left(-V \xi+\frac{\zeta}{2 \kappa}\right)\right] \exp \left(-\mathrm{i} \frac{\zeta}{2 \kappa}\right) \\
& \Theta(\xi, \zeta)=\mu \frac{\xi+V \zeta}{\sqrt{1+2 \kappa V^{2}}}, \quad \eta=\frac{2+\sigma}{\alpha}\left(\frac{\mu^{2}}{2 \sigma^{2}}\right) \Gamma, \quad \mu=\frac{\sigma \alpha}{2+\sigma} \sqrt{\frac{2(1+\sigma)}{\gamma}}
\end{aligned}
$$

The \pm sign sets the parity of the solution and $\Gamma>0$ is a free parameter. It is interesting to note that relation (6e), defining $\mu_{C R}$ for the bright soliton, coincides with expression (7d), which gives $\mu$ for the boundary soliton. The amplitude parameter $\eta$ assumes the same functional dependence in solutions (6) and (7), and these solutions also share a common formal phase factor. The width of transition region between the two plateaux of a boundary soliton, which is quantified by $1 / \mu$, decreases as the index $\sigma$ increases. This is expected intuitively since for a stationary (propagation invariant) solitary wave, an increase in the non-linearity must be accompanied by a corresponding increase in the strength of diffraction (i.e. a narrowing of the boundary-soliton width). $\quad \mu$ is determined uniquely by nominal material parameters (i.e. $\alpha, \gamma$ and $\sigma$ ). However, it should be noted that the ratio $\alpha / \sqrt{\gamma}$, which appears in Eqs. (6e) and (7d), may depend on field amplitude $E_{0}$, so that the specified value of $\mu$ for boundary solitons can actually be attained through variation in light intensity. Solution (3) has a "linked-plateau" topology in both its amplitude and intensity distribution, but there is no intrinsic phase shift across the wave profile. As such, boundary solitons play a natural role as non-linear waves that connect regions of finite- and zero-amplitude disturbance. Such waves are known in other branches of physics [3]. They arise in universal models such as the Fisher-KPP and Burgers equations and are sometimes referred to as shocks, diffusive solitons or power-balanced solitary waves.

The distinctive asymmetry of boundary solitons is manifest in the asymptotics of a solution such as $u_{-}$, where

$$
\lim _{\xi+V \zeta \rightarrow-\infty}\left|u_{-}\right| \rightarrow 0 \quad \text { and } \quad \lim _{\xi+V \zeta \rightarrow+\infty}\left|u_{-}\right| \rightarrow\left[\frac{1+\sigma}{2+\sigma}\left(\frac{\alpha}{\gamma}\right)\right]^{1 / \sigma} .
$$

These limits are reversed for the opposite-parity solution $u_{+}$. This amplitude topology differs fundamentally from that of more familiar dark solitons [8,30], where definite parity in these latter solutions leads to an intensity distribution that comprises a localized (symmetric) "grey dip" on a uniform background. One may consider, for simplicity, on-axis beams and define an inversion operation of $\xi \rightarrow-\xi$ (corresponding to reflection in the propagation axis). Under this operation, bright- and black-soliton solutions behave as $u \rightarrow u$ and $u \rightarrow-u$, respectively. On the other hand, inversion of boundary solitons carries solutions (7) into each other; that is $u_{ \pm} \rightarrow u_{\mp} \neq \mp u_{ \pm}$. Each of the above results from inversion transformation is consistent with the distinct topology of the solution concerned, and each provides 

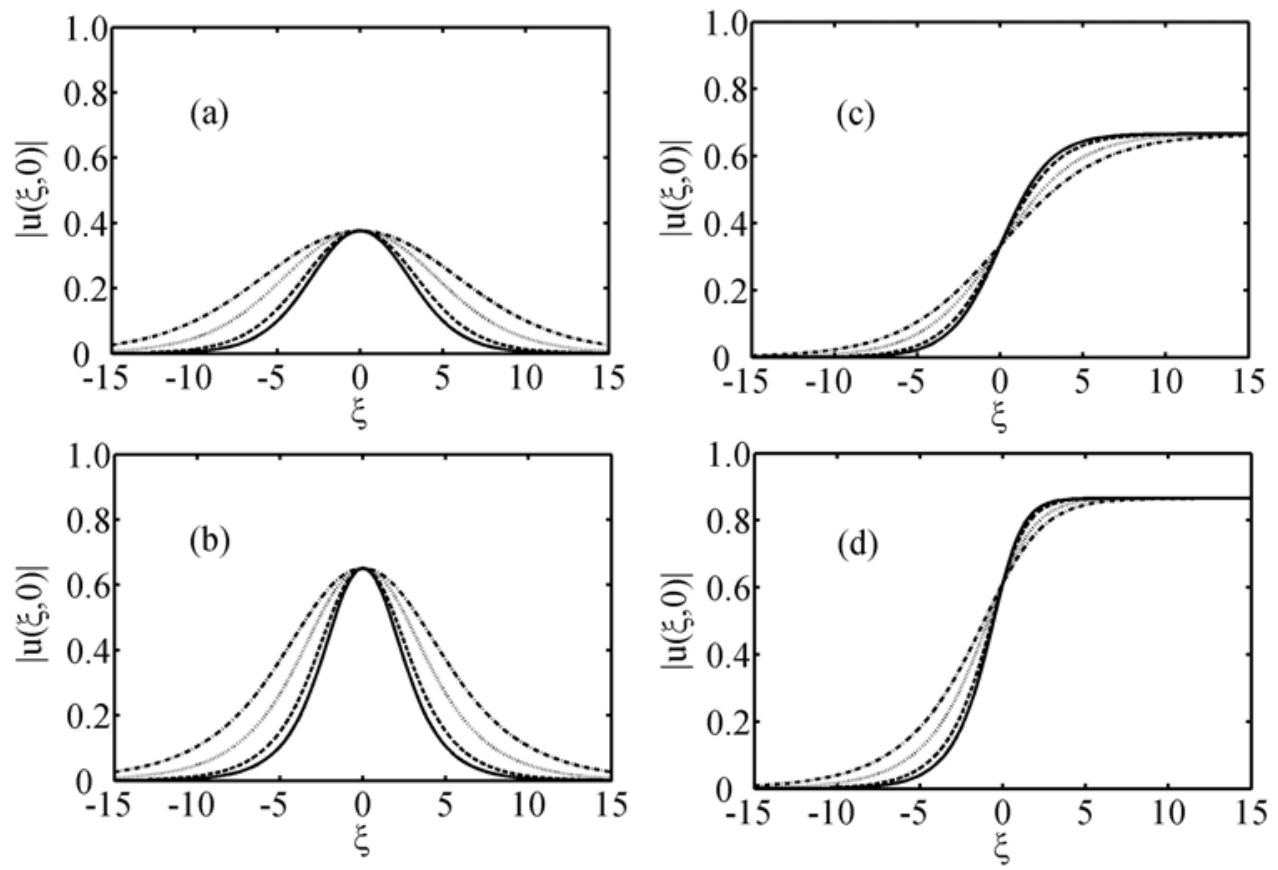

Figure 1. Angular beam broadening effect during off-axis propagation. (a) $\sigma=1$ and (b) $\sigma=2$ bright sech-type soliton solutions (6) for $\mu=0.9 \mu_{C R}$. (c) $\sigma=1$ and (d) $\sigma=2$ boundary soliton solutions (7) with $\Gamma=1$. Solid line: $\theta=0^{\circ}$ (corresponding to the paraxial solution); dashed line: $\theta=30^{\circ}$; dotted line: $\theta=50^{\circ}$; dot-dash line: $\theta=60^{\circ}$. For a non-paraxial parameter $\kappa=10^{-3}\left(\kappa=10^{-4}\right)$, these propagation angles correspond to transverse velocities $V=0 \quad(V=0), V \approx 12.91 \quad(V \approx 40.82), V \approx 26.65$ $(V \approx 84.27)$ and $V \approx 38.73(V \approx 122.47)$, respectively. Material parameters: $\alpha=\gamma=1$.

a snap-shot of the full rotational consistencies that only arise from spatial soliton representations in a Helmholtz framework. It is interesting to compare the phase topologies of bright, boundary and dark solitons. Phase-topological dark solitons can be expressed as $u(\xi, \zeta)=u_{0} D(\xi, \zeta) \exp \left[i\left(k_{\xi} \xi+k_{\zeta} \zeta\right)\right]$, where $D(\xi, \zeta)$ represents the localized solitary dip that modulates a plane wave. If the factor $D(\xi, \zeta)$ is omitted, the solution $u(\xi, \zeta)=u_{0} \exp \left[i\left(k_{\xi} \xi+k_{\zeta} \zeta\right)\right]$ still satisfies the governing equation; indeed, it is a non-linear plane-wave solution. This kind of separation does not occur for bright and boundary solitons, where the phase distribution is non-topological and spatial inhomogeneity connects to a zero background.

Solutions (6) and (7) describe bright and boundary solitons, respectively, whose transverse velocity $V$ is related to the propagation angle $\theta=\tan ^{-1}(\sqrt{2 \kappa} V)$ with respect to the reference $(z)$ axis. When $V=0$, the width of each solution is characterized by $\Lambda_{0}=1 / \mu$, while for a non-zero transverse velocity, one has that $\Lambda=\left(1+2 \kappa V^{2}\right)^{1 / 2} \Lambda_{0}$. An observer in the $(x, z)$ frame therefore 'sees' the width of the obliquely-evolving beam, through a geometrical projection [34], as increased by a factor of $\left(1+2 \kappa V^{2}\right)^{1 / 2}=\sec \theta$ relative to its on-axis value (see figure 1). The Helmholtz correction term $2 \kappa V^{2}$ is determined solely by the propagation angle $\theta$, and may be of arbitrary order. For example, one can have an off-axis regime where $2 \kappa V^{2} \gg O(1)$, even though $\kappa \ll O(1)$ is satisfied since the Helmholtz beam is broad.

Thus, when the only source of non-paraxiality is due to oblique propagation, narrow-beam models derived from single-parameter (i.e. $\kappa$-based) order-of-magnitude considerations of Maxwell's equations [23-26] are inappropriate. To emphasize the physical context of this, consider a broad optical beam propagating in a uniform medium. One has freedom to choose the orientation of the coordinate 
frame since there is no 'preferred' direction. One may align the reference $(z)$ axis along the propagation direction of the beam, or define a coordinate system where there is a finite angle between the beam's propagation direction and the $z$ axis. When narrow-beam corrections are redundant in the first frame (because the beam is broad), they are also redundant in the second frame. These two representations are, after all, of the same beam. On the other hand, when the beam propagation angle becomes arbitrarily close to $90^{\circ}$ then the Helmholtz correction term $2 \kappa V^{2}$ becomes arbitrarily large (by trigonometric identity). Accurate descriptions of off-axis evolution [27,28,30-35] and oblique interactions [29] need, instead, to respect $x-z$ symmetry.

One can recover the bright and boundary solitons of the corresponding paraxial model [16] from the (forward) Helmholtz solutions (6) and (7) by enforcing the simultaneous limits $\kappa \rightarrow 0$ (broad beams), $\kappa\left(\mu^{2} / 2 \sigma^{2}\right) \rightarrow 0$ (moderate intensities) and $\kappa V^{2} \rightarrow 0$ (axial, or near-axial, propagation). We find that bright soliton (6) becomes

$$
u(\xi, \zeta) \simeq\left[\frac{\eta}{\cosh [\mu(\xi+V \zeta)]+\Gamma}\right]^{\frac{1}{\sigma}} \exp \left[-\mathrm{i} V \xi+\frac{\mathrm{i}}{2}\left(\frac{\mu^{2}}{\sigma^{2}}-V^{2}\right) \zeta\right]
$$

while boundary soliton (7) reduces to

$$
u_{ \pm}(\xi, \zeta) \simeq\left[\frac{\eta}{\exp [ \pm \mu(\xi+V \zeta)]+\Gamma}\right]^{\frac{1}{\sigma}} \exp \left[-\mathrm{i} V \xi+\frac{\mathrm{i}}{2}\left(\frac{\mu^{2}}{\sigma^{2}}-V^{2}\right) \zeta\right]
$$

It is this triple limit that defines paraxial solitons and these conditions are captured by the single statement $\kappa\left|\partial_{\zeta \zeta} u\right| \rightarrow 0$. We stress that the paraxial solutions (8) and (9) cannot be obtained simply by setting $\kappa=0$ in the corresponding Helmholtz solutions. For solution (9), the particular case of $\sigma=2$, $\Gamma=1$ and $V=0$ (on-axis propagation) was reported many years ago by Gagnon for a cubic-quintic NLS-type equation [36]. The existence of such boundary-soliton solution families could have been inferred from noting the mathematical similarities between the (dispersive) cubic-quintic NLS model and the pinned front-bearing (diffusive) Fisher-KPP equation. Although Gagnon's paraxial boundary soliton appears to have received little subsequent attention in the literature, its fascinating delocalized character has motivated our Helmholtz generalization.

\section{Stability criteria for bright and dark solitons}

For NLS-type equations with generalized non-linearities [16], insight into the potential instability of a localized bright solution can sometimes be gained through examination of the well-known VakhitovKolokolov integral criterion [37]. The absence of unstable eigenmodes with real instability growth rate, for a solution $u=|u(\xi, \zeta ; \Omega)| \exp (i \Omega \zeta)$ that is constrained by vanishing asymptotics (i.e. $|u| \rightarrow 0$ and $\partial_{\xi} u \rightarrow 0$ as $\left.\xi \rightarrow \pm \infty\right)$, is indicated by

$$
\frac{d P(\Omega)}{d \Omega}>0, \quad \text { where } \quad P(\Omega) \equiv \int_{-\infty}^{+\infty} d \xi|u(\xi, \zeta ; \Omega)|^{2}
$$

Here, $P(\Omega)$ is the beam power and $\Omega$ is the longitudinal wavenumber. Criterion (10) turns out to be a solvability condition for a linearized eigenvalue problem $[18,38]$ and is usually valid for examining stability against sufficiently small perturbations. Enns et al. [39] showed that (10) does not guarantee the stability of bright solitons against arbitrarily-large perturbations, and that numerical analysis is essential to address the issues of about 'robust' soliton stability.

A linear-stability criterion for dark-type solitons in NLS-type systems has been established in a mathematically rigorous manner only recently (see Ref. [6] and references therein). This framework is based on a renormalized field momentum and is valid for solutions with dark soliton-type non- 
vanishing asymptotics, where as $\xi \rightarrow \pm \infty,|u| \rightarrow$ const. and $\partial_{\xi} u \rightarrow 0$. The continuous infinity of degrees of freedom associated with the plane-wave background are subtracted in a self-consistent way, so that dark solitons can be stable if

$$
\frac{d M_{R}\left(V_{0}\right)}{d V_{0}}>0, \quad \text { where } \quad M_{R}\left(V_{0}\right) \equiv \frac{i}{2} \int_{-\infty}^{+\infty} d \xi\left(u \frac{\partial u^{*}}{\partial \xi}-u^{*} \frac{\partial u}{\partial \xi}\right)\left(1-\frac{u_{0}^{2}}{|u|^{2}}\right)
$$

is the renormalized momentum and $V_{0}$ is the intrinsic velocity.

Boundary solitons (7) and (9) possess asymptotics that correspond to neither bright- nor darksoliton excitations. Firstly, the integral in Eq. (10b) is formally infinite for solution (9). We note, in passing, that the integrals in conservation laws (5) are also divergent for the Helmholtz boundary soliton (7). Secondly, the renormalization procedure for dark solitons is not appropriate because there is no infinitely-extended plane-wave background field. Since neither integral criterion quoted above can be applied, the stability of boundary solitons in non-linear optics appears to have remained an unanswered question.

\section{Stability of Helmholtz solitons}

In this Section, the stability of the boundary- and bright-soliton solutions of equation (4) is examined through numerical perturbative techniques. Consideration of linear stability analysis of plane-wave backgrounds is presented in the Appendix.

We have examined the stability of a range of Helmholtz solitons under perturbations to their angular spectrum using well-tested numerical methods [29-31,33,34]. In the course of these stability analyses, there have arisen four broad classes of behaviour in the parameters (amplitude, width and area $=$ amplitude $\times$ width) of the reshaping beam: (i) monotonically-decreasing oscillations that eventually vanish to leave a stationary state, (ii) rapid emergence of a stationary state with no accompanying oscillatory features, (iii) sustained self-oscillations that survive in the long-term evolution, or (iv) diffractive spreading toward a zero-amplitude state. Kerr bright beams [21] exhibit behaviour (i), while dark beams [22] evolve as described in (ii). We have classified these solitons as weakly- and stronglyattracting fixed-points of the system, respectively, due to their distinct rates of convergence toward stationary states. Perturbed power-law soliton beams tend to display the oscillatory behaviour described in (iii). We have classified these solution families as stable limit cycles, and have attributed the oscillatory characteristic to the excitation of an internal mode [31]. Bright solitons of a polynomial-type nonlinearity have been found to display the characteristics described as (i), (iii) and (iv), depending upon system parameters [33].

\subsection{Bright solitons}

We first examine the stability of bright solution (6) under angular perturbations. Since Helmholtz nonparaxiality requires $\kappa \ll O(1)$ and $\kappa\left(\mu^{2} / 2 \sigma^{2}\right) \ll O(1)$, the transverse phase gradient of solution (6) is well approximated by

$$
S=V \sqrt{\frac{1+4 \kappa\left(\mu^{2} / 2 \sigma^{2}\right)}{1+2 \kappa V^{2}}} \simeq \frac{V}{\sqrt{1+2 \kappa V^{2}}}=\frac{\sin \theta}{\sqrt{2 \kappa}} .
$$

An initial condition for equation (4) is then chosen to be the exact paraxial solution (8),

$$
u(\xi, 0)=\left[\frac{\eta}{\cosh (\mu \xi)+\Gamma}\right]^{\frac{1}{\sigma}} \exp \left(-i S_{0} \xi\right)
$$


By applying a rotational transformation [34] to (13) and examining the beam in the $\theta$ direction, it can be seen that the initial condition may be regarded, in this new coordinate system, as an on-axis soliton whose width has been decreased by the Helmholtz factor $\sec \theta=\left(1+2 \kappa V^{2}\right)^{1 / 2}$, where $V=S_{0}\left(1-2 \kappa S_{0}^{2}\right)^{-1 / 2}$. We present results for the non-linearity powers $\sigma=1$ (quadratic-cubic nonlinearity), 2 (cubic-quintic non-linearity), 3 and 4, where the input beams are launched at the nonparaxial angles $\theta=15^{\circ}, 30^{\circ}$ and $45^{\circ}$. For $\kappa=10^{-3}\left(\kappa=10^{-4}\right)$, these angles correspond to $S_{0} \approx 5.79$ $\left(S_{0} \approx 18.30\right), S_{0} \approx 11.18\left(S_{0} \approx 35.36\right)$ and $S_{0} \approx 15.81\left(S_{0}=50.0\right)$ respectively. The curves shown in figures 2 and 3 are universal in the sense that they hold for any combination of $\kappa$ and $V$ so long as the product $\kappa V^{2}=\frac{1}{2} \tan ^{2} \theta$ is preserved. The scalings of equation (4) are such that as $\kappa$ becomes smaller (i.e. the beam becomes broader), the length-scale of the reshaping oscillations increases in the $(x, z)$ frame since $z=\zeta / 2 \kappa k$. Without loss of generality, the material parameters are set to $\alpha=\gamma=+1$; these coefficients can be eliminated through a rescaling of equation (4), and one thus expects the same qualitative behaviour to arise for arbitrary choices of $\alpha$ and $\gamma$. This has been confirmed by direct simulation.

We begin by considering initial condition (13) with $\mu=0.9 \mu_{C R}$. Figure 2(a) shows that when $\sigma=1$, sustained self-oscillations survive in the long-term evolution of the beam parameters. The oscillation are contained within an envelope that varies quasi-periodically in the propagation direction. For $\sigma=2$ and $\sigma=3$ [figures 2(b) and 2(c), respectively], the parameters undergo monotonicallydecreasing oscillations that vanish as $\zeta \rightarrow \infty$. This behaviour is reminiscent of Kerr bright solitons [34]. For $\sigma=4$, weak and moderate perturbations have been found to give rise to monotonicallydeceasing oscillations. However, for strongly perturbed beams, self-focusing cannot balance diffractive spreading and the initially localized state diffracts and disappears as $\zeta \rightarrow \infty$ [dot-dash curve in figure 2(d)]. This type of conditional stability has been discovered for some power-law [31] and algebraic [33] solitons. Extensive numerical simulations have revealed that the global characteristic (i.e. sustained/decaying oscillations or diffractive spreading) of any reshaping beam appears to be independent of $\mu$.
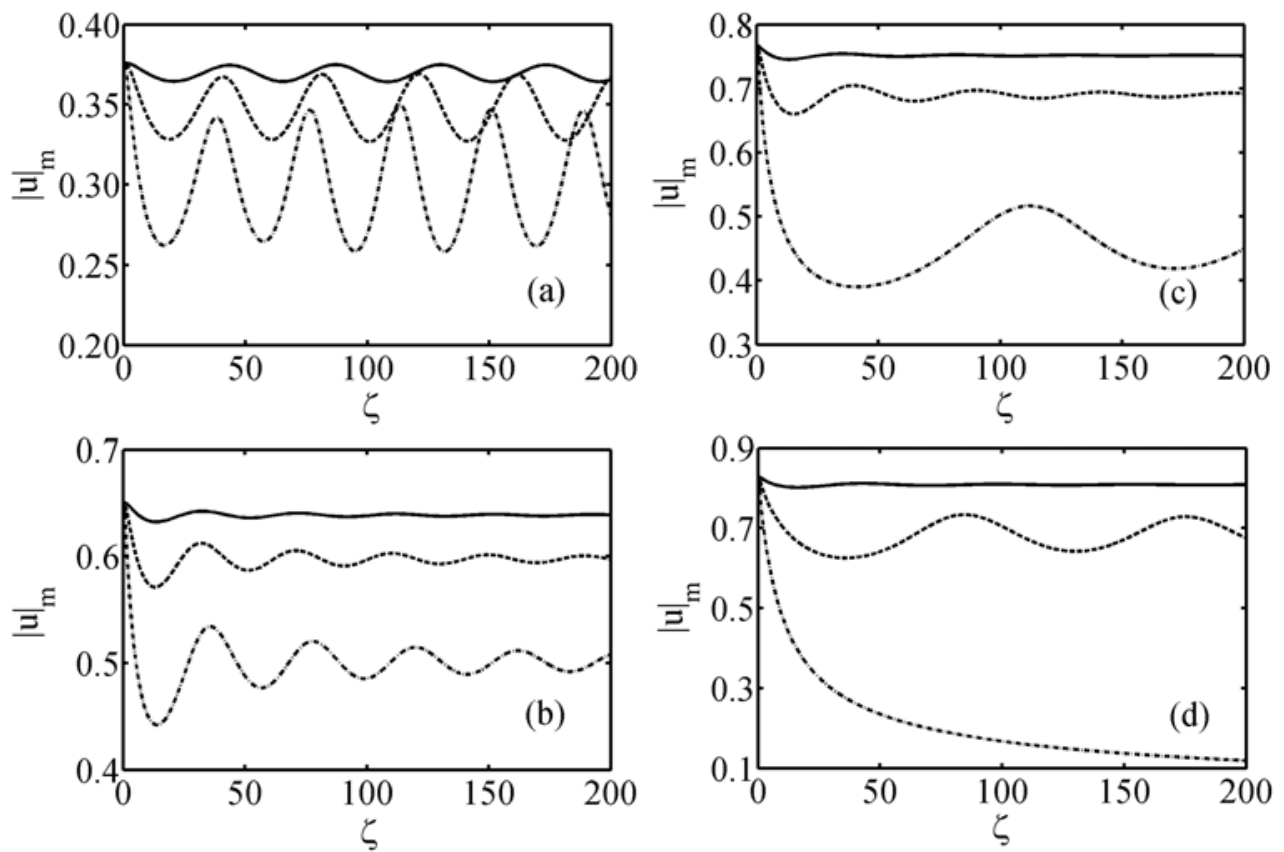

Figure 2. Evolution of the peak amplitude $|u|_{m}$ for initial condition (13) with $\mu=0.9 \mu_{C R}$ for (a) $\sigma=1$, (b) $\sigma=2$, (c) $\sigma=3$, and (d) $\sigma=4$. Solid line: $\theta=15^{\circ}$; dashed line: $\theta=30^{\circ}$; dot-dash line: $\theta=45^{\circ}$. 


\subsection{Boundary solitons}

We now choose an initial condition for equation (4) in the form of an exact paraxial boundary soliton (9),

$$
u_{-}(\xi, 0)=\left[\frac{\eta}{\exp (-\mu \xi)+1}\right]^{\frac{1}{\sigma}} \exp \left(-i S_{0} \xi\right)
$$

Figure 3 demonstrates that the width of the boundary soliton undergoes monotonically-decaying oscillations, tending toward an asymptotic linked-plateau intensity distribution that is stationary as $\zeta \rightarrow \infty$. For the cubic-quintic non-linearity $(\sigma=2)$, the asymptotic width is given precisely by $\Lambda_{\infty} \sim \mu^{-1}\left(1+2 \kappa V^{2}\right)^{1 / 2}$. For other values of $\sigma$, we find that the boundary width that emerges is slightly less than that predicted.

Numerical simulations have revealed three intriguing aspects of boundary-soliton stability. Firstly, despite its asymmetric delocalized structure, perturbed boundary-soliton initial conditions evolve toward their stationary states in a manner reminiscent of exponentially-localized Kerr solitons [34]. We suspect that this may be related to their phase distributions being non-topological. Secondly, in all our simulations, a stationary boundary soliton was found to emerge from the initial condition. Thirdly, this stationary state is reached much sooner for boundary solitons than for localized sech-type solitons (one can compare the longitudinal length scales in figures 2 and 3). These surprising results show that the new delocalized solutions (9) are generally more robust than their localized (2) counterparts. In particular, we have uncovered no evidence of the conditional stability found for $\sigma=4$ bright solitons in the corresponding boundary soliton [see figure 2(d)].
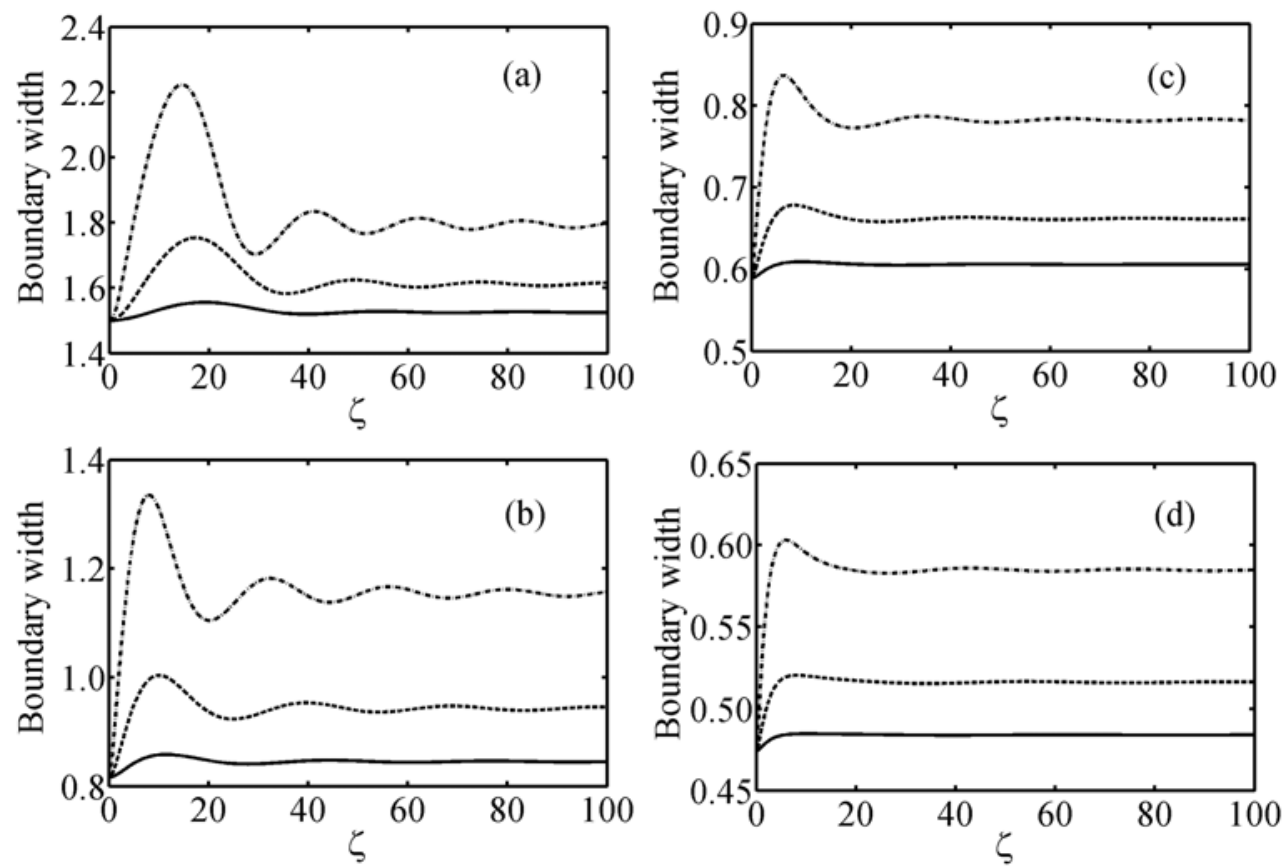

Figure 3. Evolution of the boundary-soliton width for initial condition (10), with $\Gamma=1$ and for (a) $\sigma=1$, (b) $\sigma=2$, (c) $\sigma=3$, and (d) $\sigma=4$. Solid line: $\theta=15^{\circ}$; dashed line: $\theta=30^{\circ}$; dot-dash line: $\theta=45^{\circ}$.

\subsection{Discussion}

Behaviour of dissipative non-linear dynamical systems is often analysed within a phase-space representation [18,40,41]. One can identify universal attractors (or trajectories) that appear in a particular phase plane, such as fixed points or limit cycles. Fixed points represent stationary states of the system. Trajectories that are attracted to such points may involve damped oscillatory motions, such as those shown in figures 2(b) and 2(c). Those $\theta=15^{\circ}$ solutions (solid lines) are represented in a phase space in 
figures 4(a) and 4(b). Numerical analysis reveals that boundary solitons have qualitatively similar propagation properties (see figure 3 ). It is in this sense that we classify boundary solitons, and some families (i.e. $\sigma=2$ and $\sigma=3$ ) of bright solitons, as fixed-point attractors. Sometimes, periodic and quasi-periodic trajectories arise in the evolution of beams, for example the $\sigma=1$ solution shown in figure 2(a). In this case, one finds closed-figure or nearly-closed-figure trajectories in the phase plane, as shown in figure 3(c). These phase portraits resemble classic limit-cycle orbits in non-linear dynamics [41], and this is the basis for classifying $\sigma=1$ solitons as limit-cycle attractors. Finally, one sometimes encounters trajectories that converge monotonically toward a zero-amplitude asymptotic state. This occurs with strongly-perturbed $\sigma=4$ bright solitons, as shown in figure 2(d). In this case, the initial condition may be interpreted as lying outside the soliton basin of attraction and inside that of a fixed point with zero amplitude. The $\sigma=4$ bright soliton families are, in this sense, conditionally-stable solutions.

Figure 5 illustrates what types of trajectory arise from perturbed bright solitons in a section of the $(\sigma, \theta)$ plane. Generally, one tends to find only limit-cycle behaviour in the region $0<\sigma \leq 1.5$ and fixed-point behaviour in $1.5<\sigma \leq 3$. For $\sigma>3$, the solitons tend to show conditional stability: small and moderate perturbations $\left(\theta \leq 30^{\circ}\right)$ lead to fixed-point behaviour, while larger perturbations $\left(\theta>30^{\circ}\right)$ can lead to diffractive spreading.
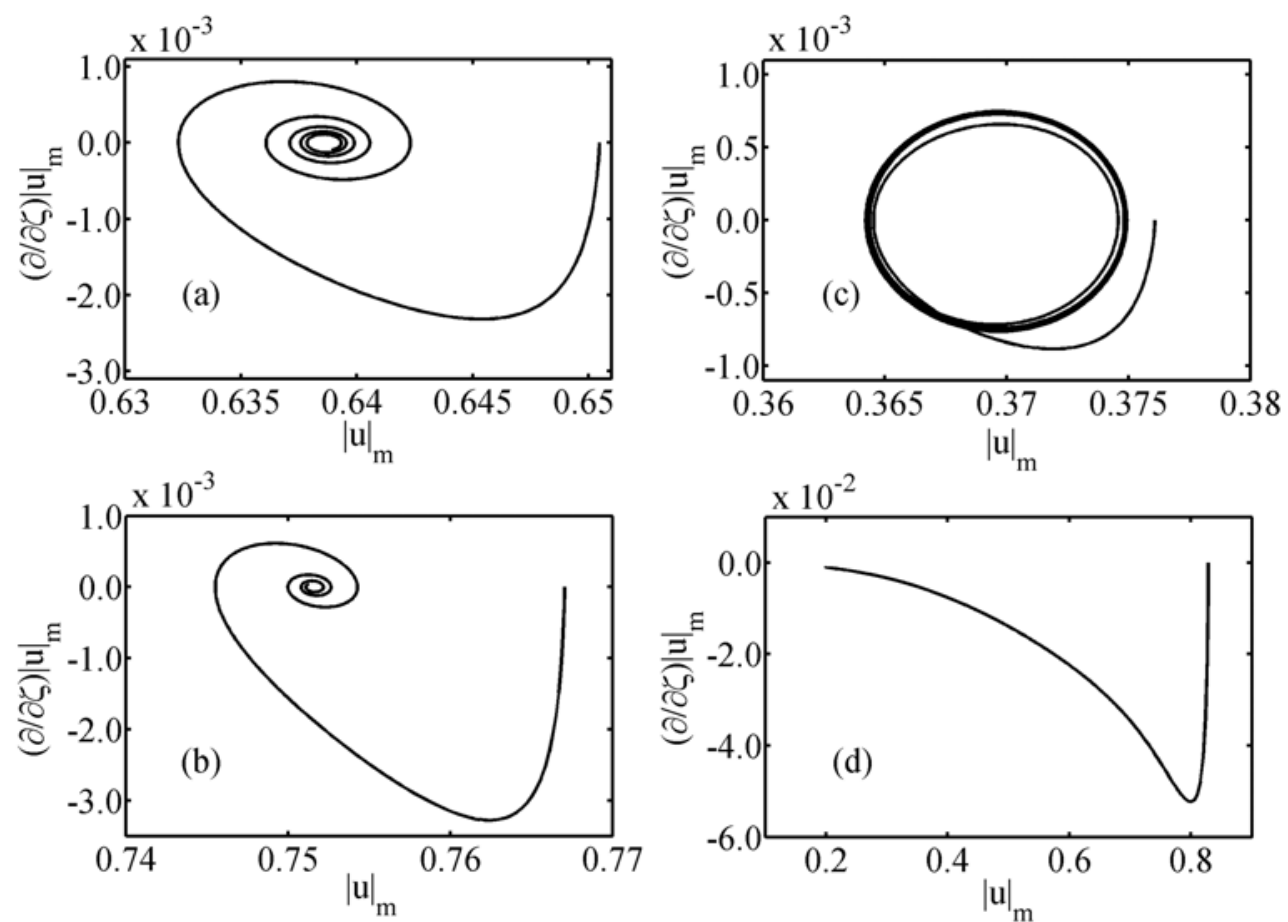

Figure 4. Phase portraits for the perturbed bright soliton (6) arising from initial condition (13). (a) and (b) correspond to the $\theta=15^{\circ}$ curves of figures 2(b) (i.e. $\sigma=2$ ) 2(c) $(\sigma=3)$, respectively. The trajectories wind asymptotically on to points in the phase plane. These points lie on the $(\partial / \partial \zeta)|u|_{m}=0$ axis (indicating that a stationary state has been reached), their position on the $|u|_{m}$ axis depends upon the perturbation $\theta$. The $\theta=15^{\circ}$ limit-cycle trajectory of figure 2(a) $(\sigma=1)$ is represented here in part (c). The trajectory in (d) corresponds to the diffracting beam of figure 2(d), where a (conditionallystable) $\sigma=4$ soliton is strongly perturbed $\left(\theta=45^{\circ}\right)$.

\section{Conclusions}

We have presented, for the first time, a novel family of boundary soliton solutions of an NLH equation with a competing polynomial-type non-linearity. The most general off-axis solution (3) was derived from an orthogonal transformation of the on-axis beam, and exhibits a range of non-trivial Helmholtztype corrections to its paraxial counterpart (9). The latter solution appears to have been reported only for the cubic-quintic class of non-linearity [36], which our more general model includes as a subset. 
Extensive numerical simulations have revealed that the stability characteristics of boundary solitons closely resemble those of exponentially-localized bright solitons (6), and we have thus classified them as stable fixed points of the system. The fixed-point and limit-cycle classifications discussed in this paper are useful because they describe qualitatively, in simple terms, how various solitary-wave solutions tend to evolve when perturbed.

The new Helmholtz boundary solitons are of fundamental mathematical and physical interest. They expand the range of known exact analytical solutions of fully second-order non-integrable wave equations. We have shown that, despite their spatially-extended structure, boundary solitons are remarkably stable against perturbations. These robust structures connect regions of finite- and zero-amplitude disturbance. This feature suggests that they also may be termed "edge solitons", since they can act as natural non-linear boundary waves at the outer limits of, e.g. optical, disturbance. Moreover, the full Helmholtz framework of their definition permits the application of these exact analytical solutions with any orientation in the waveguide plane. Finite-size effects tend to play a profound role in two-dimensional transverse pattern formation and it seems quite plausible that our Helmholtz boundary solitons, or higher-dimensional counterparts, may also find application in this important subject area [42-44].

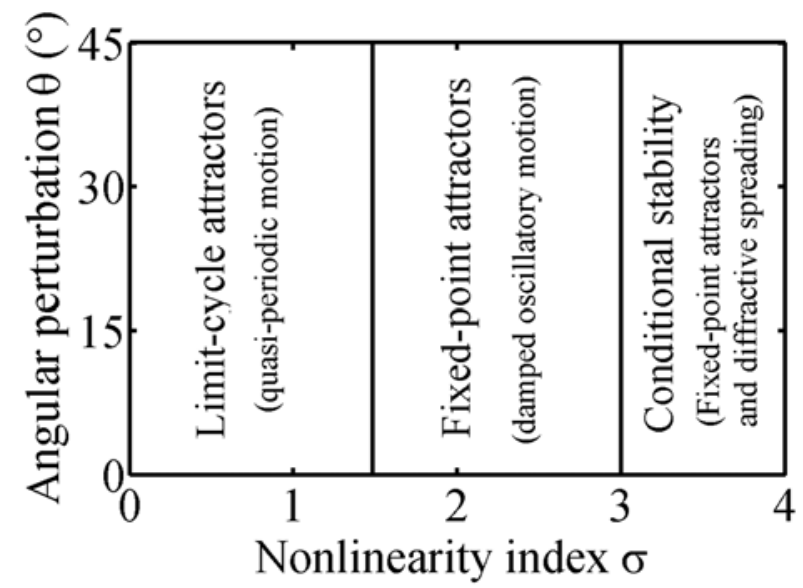

Figure 5. Schematic diagram illustrating the asymptotic behaviour of perturbed bright-solitons [initial condition (13)] in the $(\sigma, \theta)$ plane.

\section{References}

[1] Kivshar Y S and Malomed B A 1989 Rev. Mod. Phys. 61765

[2] Dodd R K, Eilbeck J C, Gibbon J D and Morris H C 1982 Solitons and Nonlinear Wave Equations (London: Academic Press)

[3] Remoissenet M 1999 Waves Called Solitons: Concepts and Experiments, ${ }^{\text {rd }}$ Ed (Berlin: Springer-Verlag)

[4] Infeld E and Rowlands G 2000 Nonlinear Waves, Solitons and Chaos, $2^{\text {nd }}$ Ed (Cambridge: C.U.P.)

[5] Kivshar Y S 1998 Opt. Quant. Electron. 30571

[6] Kivshar Y S and Luther-Davies B 1998 Phys. Rep. 29881

[7] Zakharov V E and Shabat A B 1972 Sov. Phys. JETP 3462

[8] Zakharov V E and Shabat A B 1973 Sov. Phys. JETP 37823

[9] Pushkarov K I, Pushkarov D I and Tomov I V 1979 Opt. Quant. Electron. 11479

[10] Gatz S and Herrmann J 1992 IEEE J. Quant. Elec. 281732

[11] Herrmann J 1992 Opt. Commun. 91337

[12] Kivshar Y S, Afansjev V V and Snyder A W 1996 Opt. Commun. 126348

[13] Chao W 2000 Opt. Commun. 175239

[14] Snyder A W and Mitchell D J 1993 Opt. Lett. 18101

[15] Mihalacke D, Bertolotti M and Sibilia C 1989 in Progress in Optics XXVII, E. Wolf ed. (Elsevier, Amsterdam), pp. 228-309

[16] Micallef R W, Afanasjev V V, Kivshar Y S and Love J D 1996 Phys. Rev. E 542936

[17] Hayata K and Koshiba M 1995 Phys. Rev. E 511499 
Hayata K and Koshiba M 1995 Phys Rev. E 515155

Hayata K and Koshiba M 1994 J. Opt. Soc. Am. B 112581

[18] Pelinovski D E, Afansjev V V and Kivshar Y S 1996 Phys. Rev. E 531940

[19] Wood V E, Evans E D and Kenan R P 1988 Opt. Commun. 69156

[20] Krolikowski W and Luther-Davies B 1992 Opt. Lett. 171415

Krolikowski W and Luther-Davies B 1992 Opt. Lett. 18189

[21] Lax M, Louisell W H and. McKnight W B 1975 Phys. Rev. A 111365

[22] Chi S and Guo Q 1995 Opt. Lett. 201598

[23] Ciattoni A, Di Porto P, Crosignani B and Yariv A, 2000 J. Opt. Soc. Am. B 17809

[24] Crosignani B, Yariv A and Mookherjea S 2004 Opt. Lett. 291524

[25] Ciattoni A, Crosignani B, Mookherjea S and Yariv A 2005 Opt. Lett. 30516

[26] Ciattoni A, Crosignani B, Di Porto P, Scheuer J and Yariv A 2006 Opt. Express 145517

[27] Chamorro-Posada P, McDonald G S and New G H C 2002 J. Opt. Soc. Am. B 191216

[28] Chamorro-Posada P, McDonald G S and New G H C 1998 J. Mod. Opt. 451111

[29] Chamorro-Posada P and McDonald G S 2006 Phys. Rev. E 74, 036609

[30] Chamorro-Posada P and McDonald G S 2003 Opt. Lett. 28825

[31] Christian J M, McDonald G S and Chamorro-Posada P 2006 "Helmholtz solitons for a power-law non-linearity," to be submitted

[32] Christian J M, McDonald G S and Chamorro-Posada P 2006 "Helmholtz-Manakov solitons," accepted for publication in Phys. Rev. E

[33] Christian J M 2006 "On the theory of Helmholtz solitons," PhD Thesis, University of Salford, U.K.

[34] Chamorro-Posada P, McDonald G S and New G H C 2000 J. Mod. Opt. 471877

[35] Chamorro-Posada P, McDonald G S and New G H C 2001 Opt. Commun. 1921

[36] Gagnon L 1989 J. Opt. Soc. Am. A 91477

[37] Vakhitov M G and Kolokolov A A 1973, Radiophys. Quantum Electron. 16783

[38] Laedke E W, Spatschek K H and Stenflo L 1983 J. Math. Phys. 242764

[39] Enns R H, Rangnekar S S and Kaplan A E 1987 Phys. Rev. A 35466

Enns R H., Rangnekar S S and Kaplan A E 1987 Phys. Rev. A 361270

Kaplan A E 1986 Phys. Rev. Lett. 57778

Kaplan A E 1985 Phys. Rev. Lett. 551291

[40] Zhou C, He X T and Chen S 1992 Phys. Rev. A 462277

[41] Goldstein H Classical Mechanics $19802^{\text {nd }}$ ed. Addison-Wesley, Philippines

[42] Coullet P, Riera C and Tresser C 2000 Phys. Rev. Lett. 843069

[43] Kubstrup C, Herrero H and Pérez-García C 1996 Phys. Rev. E 541560

[44] Cross M C and Hohenberg P C 1993 Rev. Mod. Phys. 65851

[45] Christian J M, McDonald G S and Chamorro-Posada P 2006 J. Phys. A: Math. Gen. 3915355

[46] Hong W-P 2002 Opt. Commun. 213173

[47] Kivshar Y S, Anderson D and Lisak M 1993 Phys. Scr. 47679 


\section{APPENDIX}

This Appendix is given in three parts. Firstly, we present general considerations of the stability of nonlinear plane-waves that propagate under the combined influence of two-dimensional diffraction and non-linear dispersion. Secondly, we detail the (long-wave) stability of plane-waves in dispersive media. Finally, we explore the consequences of the elliptic character of Helmholtz non-linear wave equations and show that any instability that appears to arise from the additional roots of the stability analysis actually corresponds to non-physical short-waves.

\section{A1. Plane-wave stability analysis}

Here, stability analysis of plane-wave solutions of the generalized NLH equation,

$$
\kappa \frac{\partial^{2} u}{\partial \zeta^{2}}+i \frac{\partial u}{\partial \zeta}+\frac{1}{2} \frac{\partial^{2} u}{\partial \xi^{2}}+f(I) u=0
$$

is considered, where $I \equiv|u|^{2}$ and $f(I)$ is an arbitrary but well-behaved function of the intensity $I$ satisfying $f(0)=0$. Equation (A1) has the obliquely-propagating plane-wave solution [45],

$$
u(\xi, \zeta)=\sqrt{I_{0}} \exp \left[\mathrm{i} k_{\xi} \xi+\mathrm{i} \sqrt{1+4 \kappa \Delta k\left(k_{\xi}^{2}, I_{0}\right)} \frac{\zeta}{2 \kappa}\right] \exp \left(-\mathrm{i} \frac{\zeta}{2 \kappa}\right),
$$

where

$$
\Delta k\left(k_{\xi}^{2}, I_{0}\right)=f\left(I_{0}\right)-\frac{1}{2} k_{\xi}^{2}
$$

that evolves in the forward direction. It is straightforward to show that the transverse wavenumber $k_{\xi}$ cannot be arbitrarily large. Instead, it has a maximum value $\left|k_{\xi}\right|_{\max }$ given by

$$
\left|k_{\xi}\right|_{\max }=\sqrt{\frac{1+4 \kappa f\left(I_{0}\right)}{2 \kappa}} \simeq \frac{1}{\sqrt{2 \kappa}},
$$

where the approximation on the far right-hand side holds since in Helmholtz non-paraxiality one has that $\kappa \ll O(1)$ (broad beams), $I_{0} \leq O(1)$ and $\kappa f\left(I_{0}\right) \ll O(1)$ (moderate intensities). Condition (A2c) corresponds to the plane-wave propagating in a direction perpendicular to the longitudinal $(z)$ direction (i.e. along the $x$ axis). Solution (A2) reduces to its paraxial counterpart

$$
u(\xi, \zeta) \simeq \sqrt{I_{0}} \exp \left[\mathrm{i} k_{\xi} \xi+\mathrm{i} \Delta k\left(k_{\xi}^{2}, I_{0}\right) \zeta\right]
$$

in the limit $\kappa \rightarrow 0$ and $\kappa \Delta k \rightarrow 0$. Paraxial theory assumes implicitly that $k_{\xi}$ is sufficiently small for the slowly-varying envelope approximation to hold (i.e. waves travel close to or along the $z$ axis), and no formal physical limit, analogous to (A2c), arises for solutions (A3). The stability of plane-wave solutions does not depend on their propagation direction, so for simplicity we perturb the on-axis solution (A2), obtained by setting $k_{\xi}=0$, according to $[46,47]$,

$$
u(\xi, \zeta)=\left[\sqrt{I_{0}}+a(\xi, \zeta)\right] \exp \left[\mathrm{i}\left(-1+\sqrt{1+4 \kappa f\left(I_{0}\right)}\right) \frac{\zeta}{2 \kappa}\right]
$$


where $a(\xi, \zeta)$ is a complex function. After substitution into equation (A1), the non-linear function is expanded using a Taylor series. Linearizing around the plane-wave solution then leads to the following equation governing evolution of the perturbation field

$$
\kappa \frac{\partial^{2} a}{\partial \zeta^{2}}+i \sqrt{1+4 \kappa f\left(I_{0}\right)} \frac{\partial a}{\partial \zeta}+\frac{1}{2} \frac{\partial^{2} a}{\partial \xi^{2}}+I_{0} f^{\prime}\left(I_{0}\right)\left(a+a^{*}\right)=0
$$

One can introduce a quite general ansatz

$$
a(\xi, \zeta)=a_{1} \exp \left[i\left(K_{\xi} \xi+K_{\zeta} \zeta\right)\right]+a_{2} \exp \left[-i\left(K_{\xi} \xi+K_{\zeta} \zeta\right)\right]
$$

that allows both forward- and backward-propagating waves and where, by convention, $K_{\xi}$ is a real parameter. Modulational instability results whenever $K_{\zeta}$ has a non-zero imaginary part. Collecting coefficients of the exponential terms and seeking non-trivial solutions gives a quartic polynomial in $K_{\zeta}$ :

$$
\kappa^{2} K_{\zeta}^{4}-\left[1+4 \kappa f\left(I_{0}\right)+2 \kappa I_{0} f^{\prime}\left(I_{0}\right)-\kappa K_{\xi}^{2}\right] K_{\zeta}^{2}+\frac{1}{2} K_{\xi}^{2}\left[\frac{1}{2} K_{\xi}^{2}-2 I_{0} f^{\prime}\left(I_{0}\right)\right]=0
$$

Setting $K_{\zeta}^{2} \equiv s$, equation (A7) assumes a quadratic form

$$
\kappa^{2} s^{2}-b\left(K_{\xi}^{2}, I_{0}\right) s+c\left(K_{\xi}^{2}, I_{0}\right)=0,
$$

where

$$
b\left(K_{\xi}^{2}, I_{0}\right) \equiv 1+4 \kappa f\left(I_{0}\right)+2 \kappa I_{0} f^{\prime}\left(I_{0}\right)-\kappa K_{\xi}^{2},
$$

and

$$
c\left(K_{\xi}^{2}, I_{0}\right) \equiv \frac{1}{2} K_{\xi}^{2}\left[\frac{1}{2} K_{\xi}^{2}-2 I_{0} f^{\prime}\left(I_{0}\right)\right] .
$$

\section{A2. Long-wave instability}

It is well-known [47] that plane-waves propagating in polynomial-type nonlinear may be subject to the spontaneous growth of transverse modulations. This (long-wave) instability has been studied in the context of paraxial models, and was found to occur when $f^{\prime}\left(I_{0}\right)>0$ and for a narrow spectral region given by $\left|K_{\xi}\right|<2\left[I_{0} f^{\prime}\left(I_{0}\right)\right]^{1 / 2}$. These conditions are dictated by equation (A8c), and a requirement of $c\left(K_{\xi}^{2}, I_{0}\right)$ negative. The predicted gain [47] of perturbations is given by $\sqrt{2}\left|K_{\xi}\right| \sqrt{2 I_{0} f^{\prime}\left(I_{0}\right)-\frac{1}{2} K_{\xi}^{2}}$. In this paper, $f(I)=\alpha I^{\sigma / 2}-\gamma I^{\sigma}$, and stability of the finite-amplitude plateau regions has been confirmed by fully non-linear numerical simulations.

\section{A3. Short-wave instability}

To examine the character of potential further instability regimes, arising from the quartic character of equation (A7), we set $K_{\zeta}=K_{R}+i K_{I}$ and seek regimes where $K_{I}$ is non-zero. Some algebra reveals that this can occur when $f^{\prime}\left(I_{0}\right)<0$, and hence when $c\left(K_{\xi}^{2}, I_{0}\right)$ is always positive. In this case,

$$
K_{I}=\operatorname{Re}\left\{ \pm \frac{1}{2 \kappa}\left[2 \kappa \sqrt{c\left(K_{\xi}^{2}, I_{0}\right)}-b\left(K_{\xi}^{2}, I_{0}\right)\right]^{1 / 2}\right\} .
$$


One can define an associated modulation growth rate [46], $g=2\left|K_{I}\right|$, given by

$$
g\left(K_{\xi}^{2}, I_{0}\right)=\frac{1}{\kappa}\left|\operatorname{Re}\left\{\left[2 \kappa \sqrt{c\left(K_{\xi}^{2}, I_{0}\right)}-b\left(K_{\xi}^{2}, I_{0}\right)\right]^{1 / 2}\right\}\right|,
$$

and the condition for instability becomes

$$
\frac{1}{2} K_{\xi}^{2}\left[\frac{1}{2} K_{\xi}^{2}-2 I_{0} f^{\prime}\left(I_{0}\right)\right]>\frac{1}{4 \kappa^{2}}\left[1+4 \kappa f\left(I_{0}\right)+2 \kappa I_{0} f^{\prime}\left(I_{0}\right)-\kappa K_{\xi}^{2}\right]^{2} .
$$

It is then straightforward to show that unstable wavenumbers are those satisfying

$$
\left|K_{\xi}\right|>\left(\frac{1}{\sqrt{2 \kappa}}\right) \frac{1+4 \kappa f\left(I_{0}\right)+2 \kappa I_{0} f^{\prime}\left(I_{0}\right)}{\left[1+4 \kappa f\left(I_{0}\right)\right]^{1 / 2}} .
$$

The expression on the right-hand side contains two factors: a geometrical term $1 / \sqrt{2 \kappa}$ and a factor that depends weakly upon the system non-linearity. In Helmholtz non-paraxiality, the second factor is $\sim O(1)$ and can be neglected. This gives the (short-wave) instability condition,

$$
\left|K_{\xi}\right|_{\min } \simeq \frac{1}{\sqrt{2 \kappa}}
$$

that depends purely on linear propagation effects, and is thus effectively independent of the nonlinearity of the system. The geometrical character of this short-wave feature is uncovered by examining the dispersion relation [35] of equation (A1). Plane-wave solutions such as (A2) lie on an ellipse in $\left(k_{\xi}, k_{\zeta}\right)$-space. The boundary between forward- and backward- propagating waves occurs at $k_{\zeta}=k_{\zeta_{\text {crit }}}=-1 / 2 \kappa$, and at this boundary $k_{\xi}= \pm\left|k_{\xi}\right|_{\max } \simeq \pm 1 / \sqrt{2 \kappa}$ (the \pm sign denotes propagation in the $\pm x$ directions, respectively). The potential short-wave instability (A12) is thus unphysical and corresponds to waves with transverse spatial wavenumbers which are greater than those that can propagate. An interpretation of this feature is that backward evanescent waves (those attenuated in the backward direction) could be mistakenly interpreted as amplified forward waves (and similarly for evanescent forward waves). 